\title{
NEW CORRECTOR SYSTEM FOR THE FERMILAB BOOSTER*
}

\author{
E.J. Prebys\#, C.C. Drennan, D.J. Harding, V. Kashikhin, J.R. Lackey, A. Makarov, W.A. Pellico \\ FNAL, Batavia, IL 6060510, U.S.A.
}

\begin{abstract}
We present an ambitious ongoing project to build and install a new corrector system in the Fermilab $8 \mathrm{GeV}$ Booster. The system consists of 48 corrector packages, each containing horizontal and vertical dipoles, normal and skew quadrupoles, and normal and skew sextupoles. Space limitations in the machine have motivated a unique design, which utilizes custom wound coils around a 12 pole laminated core. Each of the 288 discrete multipole elements in the system will have a dedicated power supply, the output current of which is controlled by an individual programmable ramp. This paper describes the physics considerations which drove the design, as well as issues in the control of the system.
\end{abstract}

\section{INTRODUCTION}

The Fermilab Booster [1] is rapid cycling synchrotron which accelerates protons from the $400 \mathrm{MeV}$ injection energy to $8 \mathrm{GeV}$ in $33 \mathrm{msec}$, at up to $15 \mathrm{~Hz}$. It has a 24 fold periodicity, with each period consisting of four combined function magnets and two straight sections, as shown in Figure 1. The "long straight" sections correspond to beta maxima in the vertical plane and beta minima in the horizontal, and the "short straight" sections are the complement.

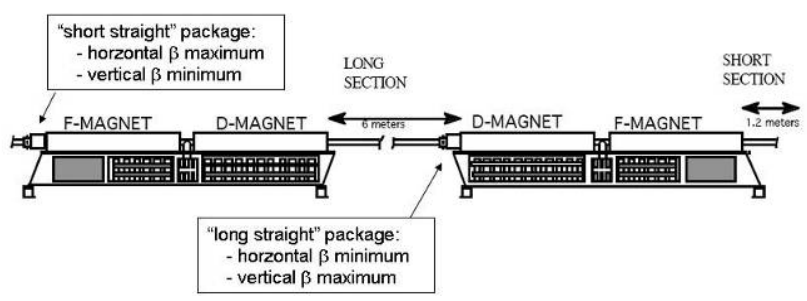

Figure 1: One of the 24 periods of the Booster

The Fermilab neutrino program places unprecedented demands Booster, which has not changed significantly since it was built more than 35 years ago. In particular, the corrector system is currently not adequate to control beam position and tune throughout the acceleration cycle, and provides limited compensation for higher order resonances.

\section{EXISTING CORRECTION SYSTEM}

The present corrector system dates back to the construction of the Booster. It comprises 48 corrector packages, each containing a horizontal and vertical dipole magnet, as well as a normal and skew quadrupoles. In general, the correctors are grouped according to the lattice functions. The 24 at the long straight sections primarily

*Work supported under DOE contract DE-AC02-76CH03000

"prebys@fnal.gov affect the vertical plane, while the 24 at the short straight sections primarily affect the horizontal. There is a two dimensional beam position monitor (BPM) at or near the location of each corrector element.

There is individual time dependent control of the dipoles corresponding to the local high beta plane (i.e. vertical at the long straights and horizontal at the short straights), while the other dipole at each section is operated DC and primarily controls the position at injection.

Beam position is controlled by adjusting currents in corrector sets according to ratios calculated to localize the beam displacement - generally "3-bumps". For the correctors with time dependent current, we implement these adjustments at discrete time breaks during the acceleration cycle. For example, we can adjust the position of the beam at a particular point at $10 \mathrm{~ms}$ into acceleration. The currents linearly interpolate between these discrete time breaks.

There is also a prototype version of a closed orbit application, which can calculate the optimum set of corrector currents to reproduce a desired orbit. The efficacy of this program is currently limited by the capabilities of the present corrector system, but it serves as a prototype for control of the new corrector system.

The quadrupole elements have individual DC levels to which common ramps are added. The ramps are implemented in four groups: long normal, long skew, short normal, and short skew. Generally speaking, the ramped terms correct for tune and coupling through the cycle, while the DC values compensate for harmonic content. To this end, a harmonic application can calculate and apply a DC offset the ith element of the form

$$
\Delta I_{n, i}=A_{n} \sin \left(n \hat{\theta}_{i}+\varphi_{n}\right)
$$

Where $n$ is any integer, $\hat{\theta}_{i}$ is the normalized phase advance of element $i$, and $A_{n}$ and $\varphi_{n}$ are "knob-able" parameters. These offsets are additive for arbitrary combinations of values of $n$.

In addition, there is a system of sextupoles at discrete locations around the ring. Sextupoles at the odd numbered short periods control horizontal chromaticity, while groups at long periods 4,10 , and 18 control vertical chromaticity. In addition, combinations of normal and skew sextupoles at long straights $4,5,6$, and 7 are configured to cancel specific third order resonances. Sextupoles are controlled in a manner similar to the quadrupoles, although the options for harmonic correction obviously more limited. 


\section{SPECIFICATIONS FOR NEW SYSTEM}

The detailed motivations and specifications for this system can be found elsewhere [2]. The correction dipole specification was driven by the requirement to maintain beam position in both planes throughout the acceleration cycle, based on observed beam motion and slewing with the existing system. The quadrupole specification was driven by the desire to maintain the tune arbitrarily close to the upper integer resonance throughout the cycle, as a possible means to mitigate the effects of space charge tune shift. The skew quadrupole strength was specified to match that of the existing system, as that has always proven adequate.

A major improvement in the new system is the introduction of sextupoles and skew sextupoles at every location. This has the advantage of being able to cancel all potential third order resonances regardless of lattice distortions. It will also eliminate the emittance increase arising from the more localized sextupole system which is in place now. The strength of the new sextupole system was specified to match the integrated strength of the existing system.

The final specifications are shown in Table 1 . The slew rates for the quadrupole and sextupole elements are motivated by the desire to change these quickly at transition.

Table 1: Corrector Magnet Specifications

\begin{tabular}{|c|c|c|}
\hline Type & $\begin{array}{c}\text { Integrated } \\
\text { field/gradient }\end{array}$ & Max slew rate \\
\hline Horizontal dipole & $0.009 \mathrm{~T}-\mathrm{m}$ & $3.24 \mathrm{~T}-\mathrm{m} / \mathrm{s}$ \\
\hline Vertical dipole & $0.015 \mathrm{~T}-\mathrm{m}$ & $3.24 \mathrm{~T}-\mathrm{m} / \mathrm{s}$ \\
\hline Normal quadrupole & $0.16 \mathrm{~T}$ & $88 \mathrm{~T} / \mathrm{s}$ \\
\hline Skew quadrupole & $0.008 \mathrm{~T}$ & $0.8 \mathrm{~T} / \mathrm{s}$ \\
\hline Normal sextupole & $1.41 \mathrm{~T} / \mathrm{m}$ & $2350 \mathrm{~T} / \mathrm{m} / \mathrm{s}$ \\
\hline Skew sextupole & $1.41 \mathrm{~T} / \mathrm{m}$ & $2350 \mathrm{~T} / \mathrm{m} / \mathrm{s}$ \\
\hline
\end{tabular}

\section{MAGNET DESIGN}

The details of the magnet design are discussed elsewhere [3]. The field specifications and space limitations within the Booster drove a unique design, based on laminated cores and twelve individual poles, as shown in Figure 2. We considered powering each of the twelve coils independently, but it was felt that this raised too many complications with regards to control. In the end, therefore, the magnets were wound with in such a way as to produce the six desired multipoles.

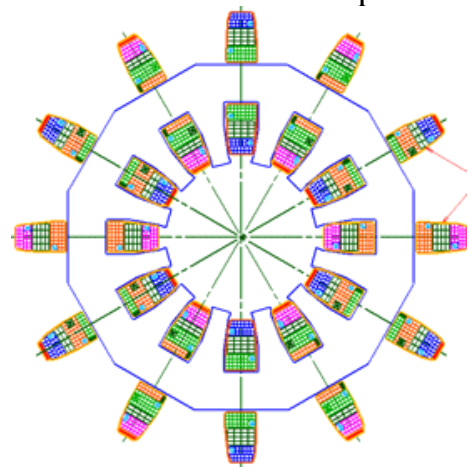

Figure 2: Coil arrangement in new correctors
In order to allow the maximum length for the magnets, BPM's have been integrated into the corrector elements.

\section{IMPLEMENTATION AND CONTROL}

The details of the corrector fabrication, testing, installation, power supplies and control are described in other contributions to this conference [4][5][6]. Each of the six elements in each of the 48 corrector packages will have a dedicated power supply. The level of each of these supplies will be individually controlled by a CAMAC module known as a "C473" [7]. This is an extremely versatile module, but the key features for our application are:

- It produces an arbitrary, time dependent ramp, synchronized with beam injection.

- $\quad$ One can optionally add a programmable offset to this ramp.

- An additional offset may be added via an external analog input.

- All of these functions can be specified differently for different types of Booster cycles, within the Fermilab event clock protocol.

The DC offset capability is intended to emulate a functionality currently implemented at the hardware level in the switching amps.

The corrector installation will occur in two stages. In the fall of 2007, the 24 corrector packages in the long straight sections will be installed, leaving the original correctors at the short straight sections. The remaining corrector packages will be installed in summer, 2008.

\section{INITIAL OPERATION}

This project represents a significant modification to the Booster. Because of the imperative of accelerator operation, it's vital that the Booster return to acceptable levels of performance as quickly as possible. To this end, we will initially configure the system such that it can be controlled in a manner that closely parallels the existing system. This is also necessary because we will initially have only half the correctors in place.

Even with this somewhat limited implementation, it is expected that the superior strength and slew rate specifications of the new system will quickly result in improved performance.

\section{Dipole Control}

Except for the increased strength, the only significant difference in the operation of the new corrector dipoles will be that all dipoles will now have time dependent, ramped control, rather than just the half that are at high beta regions. In keeping with our philosophy, we will initially operate the low beta correctors in a DC mode while operating the high beta correctors more or less as with the existing system. 


\section{Quadrupole Control}

In the case of the quadrupoles, we will now have individual ramped control of all elements; however, we do not yet have an algorithm to take full advantage of this. For that reason, we will group the modules in fours sets of 24, as they are now (long normal, long skew, short normal, and short skew), and a common ramp to all of the correctors in each set. Each element will also have an individual DC offset programmed according to harmonic weighting, or set of harmonic weightings. This will be implemented via the C473 module in a manner which emulates the current hardware configuration.

\section{Sextupole Control}

The existing limited sextupole system will be left in place, so that we can initially operate in the same mode that we do now. As we bring the new sextupoles into use, we will initially control them in exactly the same way we control the quadrupoles; namely, the time dependent portion will be controlled in four groups, while each element will have an individual DC offset determined by harmonic correction, utilizing the algorithm described in equation (1).

\section{DESIGN OPERATION}

It will be challenging to take advantage of all of the capabilities of the new corrector system. New modes of operation will be adiabatically introduced to minimize the disruption of normal operation. Clearly, the full potential of the system will only be realized after all of the corrector packages are installed.

\section{Dipole Control}

The new corrector system will have enough strength to fully control the beam position throughout the acceleration cycle. An application is being developed which will compare the beam orbit, as measured with the BPM system, to a "desired orbit", and calculate the necessary trim values to reproduce this orbit as a function of time in the acceleration cycle. This application is being commissioned with the existing corrector system, and should be ready for use very quickly after the new corrector system is operational.

\section{Quadrupole Control}

The new quadrupole system will be capable of controlling the tune throughout the cycle. This could potentially enable a feed-forward application to measure the tune on one cycle, and calculate the corrector values to correct to the desired tune on subsequent cycles.

In addition, because quadrupole elements will have individual ramped control, it will be possible to evolve the harmonic corrections during the acceleration cycle, in a manner which is not possible at the moment. The details of this correction and its operational interface are still in the conceptual stage.

\section{Sextupole Control}

The sextupoles of the new system will have roughly the same integrated strength as the existing system, and the chromaticity will likely be controlled in much the same way it is now.

As stated in the previous section, the complete sextupole coverage will allow arbitrary harmonic correction, just as with the quadrupole system. As with the quadrupole system, we foresee using the individual ramped control to evolve the harmonic correction through the acceleration cycle. The control interface should be a straightforward extension of what is implemented for the quadrupole system.

\section{CONCLUSION}

The new corrector system which will soon be implemented in the Fermilab Booster will represent a significant improvement, even when operated in a mode which emulates the current system. The development of applications to fully exploit the capabilities of the new system will present an ongoing challenge over the next few years.

\section{REFERENCES}

[1] E. L. Hubbard, et al, "Booster Synchrotron”, FERMILAB-TM-405 (1973). See also: B. Worthel, "Booster Rookie Book”, Fermilab BEAMS-DOC-1022-V1 (1998)

[2] E.J. Prebys, et al., "Booster Corrector System Specification”, Fermilab Beams-doc-1881-v5, (2006)

[3] V.S. Kashikhin, et al., "A New Correction Magnet Package for the Fermilab Booster Synchrotron," PAC’05, May 2005

[4] D.J. Harding, et al, "Design and Fabrication of a Multi-element Corrector Magnet for the Fermilab Booster”, MOPAS006, PAC '07 (2007)

[5] G. Velev, et al, "Using a Slowly Rotating Coil System for AC Field Measurements of Fermilab Booster Correctors”, MOPAS021, PAC '07 (2007)

[6] C. Drennan, et al., "System Overview for the MultiElement Corrector Magnets and Controls for the Fermilab Booster”, MOPAS005, PAC '07 (2007)

[7] D. McArthur, "CAMAC 473 Quad Ramp Controller Rev. 2.0”, Fermilab BEAMS-DOC-2361-V3 (2006) 